\title{
INITIAL EXPERIENCE OF SURGICAL MICROWAVE TISSUE PRECOAGULATION IN LIVER RESECTION FOR HEPATOCELLULAR CARCINOMA IN CIRRHOTIC LIVER
}

\author{
By
}

A. ABDELRAOUF ${ }^{1^{*}}$, H. HAMDY' H.M. EL ERIAN $^{3}$, M. ELSEBAE ${ }^{2}$, S. TAHA ${ }^{1}$, H. E. ELSHAFEY ${ }^{1}$, S. ISMAIL ${ }^{1}$ and M. HASSANY ${ }^{1}$

Department of Hepato-Bilio-Pancreatic Surgery ${ }^{1}$, National Hepatology and Tropical

Medicine Research Institute ${ }^{1}$, Cairo, National Institute of Endocrinology ${ }^{3}$, National Institute of Diabetes \& Endocrinology ${ }^{3}$, Cairo and Department of General Surgery ${ }^{2}$, Theodor Bilharz Research Institute ${ }^{2}$, Giza 12411, Egypt

( ${ }^{*}$ Correspondence: Fax:00201227943873 Email:abdelraoufamr@yahoo.fr :abdelraoufamr@gmail.com

\begin{abstract}
Surgical hepatic resection has been considered as the first-line treatment which is most effective and radical treatment for HCC, however, HCC is usually associated with poor liver function owing to chronic hepatitis or liver cirrhosis. Techniques that can eradicate the tumor and also preserve liver function are needed. Moreover, hepatic resection, in the presence of cirrhosis, raises special problem of high risk as hemorrhage and liver failure, thus, good clinical results can only be achieved by minimizing operative blood loss, time of the intervention as well as the hepatic reserve. The tremendous progress in microwave technology has recently attracted considerable attention. This study evaluated the feasibility of this new liver transection technique demonstrating the high performance of this procedure, the accuracy in terms of squeeze effect on veins and portal branch and in terms of reducing the intra operative blood loss, and minimizing the operative time for safe hepatectomy.

Twenty-six consecutive patients a first-time diagnosis of hepatocellular carcinoma (HCC) on top of liver cirrhosis were recruited for the study, from August 2011 to January 2013.All patients were subjected to full clinical examination, laboratory investigations, abdomen ultrasound (U/S), triphasic computed tomographic liver scan (CT) and dynamic magnetic resonance imaging (MRI) in some doubtful cases. Inclusion requirements were presence of resectable disease without vascular invasion or extrahepatic spread at imaging, Child-Pugh class A \& B (Score 7) liver cirrhosis, (INR) $<1$.6or platelet count $>60000 / \mathrm{mm}^{3}$ with no previous treatment. Patients were treated by applying pre-coagulation of the liver transection lines using microwave probe positioned in parallel to the line of resection by open approach after intraoperative U/S assessment for localization of the tumor and line of resection.

The procedures were performed under general anesthesia. Mobilization of the liver was not necessary to be done in all cases. Intra-operative ultrasound (Aloka, Tokyo, Japan) was used to exclude lesions not detected pre-operatively (operative surprise), to define the location, number and size of the tumor and to identify large intra-hepatic large vascular and biliary structures, and guide insertion of the microwave applicator. Intraoperative ultrasound was used again, to assess that the line of resection was done. Primary endpoints documented were total operative time, time for liver parenchyma transection, intraoperative blood loss and blood transfusion requirements. Secondary endpoints included postoperative complications, mortality and intensive care unit (ICU) together with hospital stay.
\end{abstract}

Key wards: Microwave - Hepatectomy - liver - Cirrhosis - Hepatocellular carcinoma

\section{Introduction}

Hepatocellular carcinoma (HCC) is the fifth most common cancer and the third most common cause of the cancer death in
Egypt (Nasr et al, 2014). Hepatocellular carcinoma (HCC) therapies with curative intention involve liver transplantation, surgical resection and ablation techniques (Min et al, 2014). Surgical Hepatic resection has been considered as the first-line treatment 
which is most effective and radical treatment however, HCC is usually associated with poor liver function owing to chronic hepatitis or liver cirrhosis (Abdel-Wahab et al, 2013). Therefore, techniques that can eradicate the tumor and also preserve liver function are needed. Moreover, hepatic resection, in the presence of cirrhosis, raises special problem of high risk as hemorrhage and liver failure (González and Figueras, 2009). Thus, good clinical results can only be achieved by minimizing operative blood loss, decreasing the operative time and with acceptable parenchymal reserve. The tremendous progress in microwave technology has recently attracted considerable attention. A single microwave antenna operating at $2.45 \mathrm{GHz}$ for ablation can create a $4 \times 6 \mathrm{~cm}$ ablation in 4 minutes and a $5 \times 7$ in 8 minutes in the protocol of ablation, while in the protocol of cutting the coagulation process takes between 1-2 minutes to have a coagulation distance of $2-3 \mathrm{~cm}$. This technique is much faster and creates a larger coagulation zone (Jagad et al, 2007; Bhardwaj et al, 2010; Christian et al, 2011). Microwave ablation (MWA) can be performed in cirrhotic patients with HCC with acceptable morbidity and low recurrence rates; however, local recurrence remains a concern. The microwave tissue coagulator has been designed to reduce bleeding from the liver parenchyma by coagulating the tissue before liver transection. Liver pre-coagulation with microwave technology is a novel and efficient technique which allows nonanatomical resection to be performed safely and easily, and helps prevent intrahepatic metastasis via portal flow during transection with minimal morbidity and mortality for liver transection (Tanaka et al, 2008; Reuter and Martin, 2009; Eltawil et al, 2010; Imura et al, 2012). Here the present authors describe the treatment outcomes achieved at our institutions for intraoperative use of the microwave tissue pre-coagulation in hepatic resection as part of our strategy for the treatment of HCC.

\section{Patients, Materials and methods}

Twenty-six consecutive patients a firsttime diagnosis of hepatocellular carcinoma (HCC) on top of liver cirrhosis were recruited for the study in the department of Hepatobiliary surgery inthree centers (National Hepatology and Tropical Medicine Reasearch Institute (NHIMRI), Mokattam Insurance Hospital and Dar El-Chefa Hospital) from August 2011 to January 2013.This was a prospective study which was approved by the Ethical Committee of National Hepatology and Tropical Medicine Research Institute (NHTMRI). Written informed consent was obtained from each patient before treatment. The diagnosis was based on reliable clinical criteria fulfilling association with viral hepatitis or liver cirrhosis, typical imaging findings, raised HCC-related tumor markersnamely (alpha-fetoprotein AFP). In suspicious cases histological confirmation by tumor tissue biopsy is needed, according to the Barcelona criteria of the European Association for the Study of the Liver (Iannitti et al, 2007).

All patients were subjected to full clinical examination, laboratory investigations for blood picture, coagulation profile, liver and kidney functions testing, computed tomographic scan of the chest (CT), abdomen ultrasound, triphasic computed tomographic scan of the liver (CT), Dynamic MRI in some doubtful cases and upper GIT endoscopy for verification of oesophageal varices and banding if Grade III or IV. The location of the HCC was evaluated with abdominal ultrasonography (US) confirmed with triphasic computed tomographic scan of the liver (CT). General requirements for inclusion in our study were presence of resectable disease, without vascular invasion or extrahepatic spread at imaging, Child-Pugh class $A$ and $B$ (Score 7) liver cirrhosis, (INR) < 1.6or platelet count $>60000 / \mathrm{mm}^{3}$ with no previous treatment. Patients requiring major hepatic resection ( $>$ three segments) were excluded from this study. Any coagulopathy was corrected before the procedure. Patients 
were treated by applying pre-coagulation of the liver transection lines using microwave probe positioned parallel to the line of resection by open approach after intra-operative $\mathrm{U} / \mathrm{S}$ assessment for localization of the tumor and line of resection.

\section{Operative Procedure}

The procedures were performed under general anesthesia. Prophylactic antibiotics were routinely administered. An open laparotomy was done via a right subcostal incision with possible upper midline extension if necessary or with a left subcostal extension in a chevron fashion for the resection of the left lobe tumor. The peritoneal cavity was thoroughly explored to exclude extrahepatic metastases, and aspiration of any freely intra-peritoneal fluid present for histopathological assessment. Mobilization of the liver was not necessary to be done in all cases. Intra-operative ultrasound (Aloka, Tokyo, Japan) was used to exclude lesions not detected pre-operatively (operative surprise), to define the location, number, size of the tumor, to identify large intra-hepatic large vascular and biliary structures and guide insertion of the microwave applicator. To prevent thermal injury, surrounding organs were isolated by wet towels. Microwave generator in this study was (Model HS Hospital Service S.p.A. Italy). This device emits microwaves of $2.45 \mathrm{GHz}$ in frequency and $12 \mathrm{~cm}$ in wavelength. Its maximum output is 150 watts. Microwave assisted liver resection was performed using a probe of 16 gauge diameter antennas of $19 \mathrm{~cm}$ length. Intraoperative ultrasound was used again, to assess that the line of resection was done. Then, Glisson's capsule was incised with monopolar diathermy, (Fig. I), and probe was introduced perpendicularly into the liver parenchyma (Fig. II) and the coagulation started as soon as the needle is inserted into the parenchyma. The antenna was progressively inserted into the parenchyma with 1-2 minutes intervals (time for coagulation process), when usually the color of the liver parenchyma has been changed due to com- plete coagulation of resection line, then the procedure is repeated all through the line of resection till the whole resection area is coagulated with the safety margin and the tumor was separated with a coagulation area of the parenchymal tissue around (Figure III), the liver parenchyma is dissected by sharp blade or using the Monopolar diathermy between the lesion and the remaining liver leaving 2-3 cm safe oncological margin(Figure IV). For patients who have had two tumors; the largest tumor was resected as described above meanwhile; the smaller tumor was totally ablated by the same microwave technology. Resected specimens were sent for histopathological examination for depth of tissue coagulation along the tumor-negative transection margin (Fig. V). Primary endpoints documented were total operative time, time for liver parenchyma transection, intraoperative blood loss and blood transfusion requirements. Secondary endpoints included postoperative complications, mortality and intensive care unit (ICU) together with hospital stay. In all patients, biochemical liver function tests were monitored before and after resection. Values expressed as means $\pm \mathrm{SD}$. A paired $t$ test was used to compare liver function tests before and after resection. $P<.05$ was considered significant, while other values were expressed as number percentage or as median Postoperative follow-up was carried out in the early postoperative D1, D2, D3, D7, D14 (where $\mathrm{D}=$ day) by liver function, C.B.C and renal profile, coagulation profile and chest X-ray, together with U/S at D1, D3 \& D14, then follow-up was continued after a month, then every 3 months during the following one to two years by laboratory investigations for liver function tests, serum AFP, chest Xray, U/S and triphasic computed tomographic scan of the liver (CT). The U/S and CT were evaluated by a radiologist experienced in liver radiology. Any intra- or extrahepatic recurrence was documented. The main outcome measures were operative mortality, morbidity and recurrence rate. The 
date of disease recurrence was dictated by the first imaging study showing recurrence. Local recurrence was defined as disease occurring adjacent to the surgical site, whereas remote recurrence was defined as intrahepatic tumor occurring at a distant site. Multifocal recurrence was defined as multiple intrahepatic tumors at distant sites.

\section{Results}

The patients were 19 men and 7 women with a median age of 53 (range, 42-68) years. All patients were hepatitis $C$ positive. Histological findings revealed that all patients had HCC on top of liver cirrhosis. Six patients belonged to Child's B staging (Score 7), while the rest were Child's stage A. The median tumor diameter was $3.8 \mathrm{~cm}$ (range, $2-7 \mathrm{~cm}$ ). 23patients had solitary tumors, and three patients had two tumors, accidently discovered during intra-operative $\mathrm{U} / \mathrm{S}$ assessment (Tab.1). All the procedures were completed as planned. Limited resection was performed in five patients, subsegmentectomy in eighteen patients, and segmentectomy in three patients. The median duration of the operation was 118 (range, 65-250) minutes with a median resection time of 45 (range 30-80) minutes. The average time taken to coagulate the anticipated liver transection plane was less than $15 \mathrm{~min}$. The median blood loss for resection was 165 (range, 100-750) $\mathrm{ml}$. Intraoperative blood transfusion was required in only one Child's B patient. There was no postoperative mortality. The median postoperative hospital stay was 6 (range, 2-13) days. Three Patients were admitted in ICU for 1-2 days. Two patients stayed in ICU $\geq 2$ days. All patients had a postoperative increase of liver enzymes and serum bilirubin level which normalized within 7-14 days (Tab. 2). Complications occurred in $20(76.9 \%)$ patients, who were fever in 18 patients and wound hematoma in two patients. Histopathology revealed that the depth of tissue coagulation was $3-5 \mathrm{~mm}$. It provided an additional tumor-negative margin at the resection border. The median follow-up of patients was 7 (range, 4-12) months, and their treatment outcomes are summarized in (Tab. 3). During follow up, recurrent tumors were noted in two patients $(7.69 \%)$ in the first six months, while recurrence in 15 patients was in two year follow up. Recurrent tumors were all remote recurrence (distant recurrence of the same organ). All patients survived until the time of censorship.

\section{Discussion}

The major technical difficulty in liver resection for cirrhotic patients is related to a higher risk of bleeding because of the presence of portal hypertension with portosystemic collaterals at the back of the liver, and coagulopathy as a result of compromised liver function or thrombocytopenia in patients with hypersplenism (Tarantino et al, 2009). Furthermore, maintaining adequate liver function regardless of whether the resection is anatomic or not may be of greater importance. No superiority was seen in survival and early tumor recurrence when HCC was treated by anatomic resection (Teoh and Farrell, 2003). But, small, non-anatomical resection causes more bleeding. Techniques for liver resection often require hepatic inflow occlusion (Pringle's maneuver) during transection of liver parenchyma (Matsumata et al, 1991). Besides, clamping of hepatic pedicles increases the potential risk of liver dysfunction from ischemia-reperfusion injury particularly in patients with underlying chronic liver disease (15, Pawella et al, 2014). The use of the microwave tissue coagulator has been found to minimize blood loss. Moreover, because of the more or less bloodless intrahepatic surgical field, liver transection could be carried out safer, faster and easier (Huang et al, 2009; Percivale et al, 2012; Habib et al, 2012).

The study described initial experience of that innovative technique involving the use of intra-operative microwave tissue precoagulation in hepatic resection as part of our strategy for the treatment of HCC. It was found to offer bloodless liver resection field without the use of sutures, surgical knots, 
clips, glue or hepatic pedicle clamp. When this procedure is used carefully, the surgeon can perform a liver resection with minimal blood loss. Intraoperative blood transfusion was required in only one of twenty-six patients operated upon by hepatic resection in the present study. Also, the technique reduces the anesthetic and operative time. There was no postoperative mortality and the morbidity is encountered is acceptable because of the smaller surgical insult to the patient.

Table I: Demographic and clinical characteristics of patients

\begin{tabular}{|c|c|}
\hline Item & Variable \\
\hline Male: Female ratio $* \mathrm{~N}(\%)$ & $19(73 \%): 7(27 \%)$ \\
\hline Child-Pugh class* & $20(76.9 \%)$ \\
A & $6(23.1 \%)$ \\
B & $0(0 \%)$ \\
\hline Tumor size* & \\
$2-3 \mathrm{~cm}$ & $7(29.3 \%)$ \\
$3-5 \mathrm{~cm}$ & $11(42.3 \%)$ \\
$\geq 5 \mathrm{~cm}$ & $8(30.6 \%)$ \\
\hline Tumor nodularity* Uninodular & $26(100 \%)$ \\
\hline Tumor localization* & $22(84.6 \%)$ \\
Right lobe & $3(11.5 \%)$ \\
Left lobe & $1(3.8 \%)$ \\
Bilobar & $586(4.0-1295.6)$ \\
\hline Serum AFP** (ng/ml) &
\end{tabular}

*Qualitative variables expressed as number (\%), **quantitative variables expressed as median.

Table 2: Liver functions tests as mean \pm SD before and after resection

\begin{tabular}{|l|c|c|c|c|}
\hline \multirow{2}{*}{ Characteristics } & & \multicolumn{3}{|c|}{ Postoperative assessments } \\
\cline { 2 - 5 } & Pre-Resection & $1^{\text {st }}$ Day & $7^{\text {th }}$ Day & $14^{\text {th }}$ Day \\
\hline S. albumin $(\mathrm{g} / \mathrm{dl})$ & $3.72 \pm 0.03$ & $2.78 \pm 0.13$ & $3.02 \pm 0.23$ & $3.2 \pm 0.3$ \\
\hline T. bilirubin $(\mathrm{mg} / \mathrm{dl})$ & $1.03 \pm 0.79$ & $2.03 \pm 0.04$ & $1.06 \pm 0.39$ & $0.9 \pm 0.1$ \\
\hline AST (IU/L) & $26.5 \pm 10.1$ & $115.8 \pm 10.10$ & $47.33 \pm 1.11$ & $31 \pm 3$ \\
\hline ALT (IU/L) & $31.2 \pm 4.3$ & $263 \pm 0.81$ & $78.34 \pm 0.62$ & $27 \pm 3$ \\
\hline
\end{tabular}

Table 3: Operative, Postoperative and Follow Up Data

\begin{tabular}{|l|c|}
\hline Median blood loss, ml (range) & $165(100-750)$ \\
\hline Median transection time, min (range) & $45(30-80)$ \\
\hline Median total operation time, min(range) & $118(65-250)$ \\
\hline Median hospital stay, days & $6(2-13)$ \\
\hline Patients staying in ICU for $\geq 2$ days, $n$ & 2 \\
\hline Complications & $18(69 \%)$ \\
Fever & $2(7.69 \%)$ \\
Wound hematomoa & 2 \\
\hline Intrahepatic recurrence: Remote (away from resection margin) & \\
\hline
\end{tabular}

Qualitative variables are expressed as number (percentage).

This coincides with literature review that Child-Pugh grading, operating time, postoperative length of stay, and preoperative serum albumin level were independent predictors of morbidity in hepatectomized cases (Gaujoux et al, 2011). In this small experi- ence, the technique is easy to teach, and surgeons with a good knowledge of liver anatomy can apply it to non -anatomical as well as segmental resections although the technique has not yet been used for a major liver 
resection. It may encourage surgeons to perliver surgery as a "safe" therapeutic modality in the management of liver tumors. This technique represents a considerable step in making laparoscopic liver resection safer and more feasible for many liver surgeons (Belli et al, 2009).

From the oncological point of view, nonanatomical liver sparing resection has the advantage of saving as much of liver parenchyma as possible in cirrhosis patients considering that anatomical resection was not found more advantageous in terms of tumor recurrence or survival in HCC patients (Huang et al, 2014). Moreover, microwave tissue pre-coagulation in hepatic resection lines helps prevent intrahepatic metastasis via portal flow due to tissue shrinkage and dissection with permanent closure of vascular and biliary branches. The depth of tissue coagulation of 3-5 $\mathrm{mm}$ provides an additional tumor-negative margin at the resection border. This technique also enables the surgeons to deal with accidently discovered tumors intra-operatively (operative surprise), by ablation in the same session during hepatectomy.

\section{Conclusion}

The present results showed that surgical microwave tissue pre-coagulation in liver resection for hepatocellular carcinoma in cirrhotic liver is safe and effective in reducing the blood loss and operative time, preserving as much parenchyma as possible in surgical treatment of HCC, and enables ablation intra-operatively. It achieves an acceptable recurrence rate.Further studies are needed to confirm this preliminary data to extend surgical indication to major hepatic resection and longer follow-up is required to determine the long-term outcome of this new modality.

\section{References}

Abdel-Wahab, M, Sultan, AM, Fathy, OM, Salah, T, Elshobary, MM, et al, 2013: Factors affecting recurrence and survival after living donor liver transplantation for hepatocellular carcinoma. Hepatogastroenterology 60, 128: 1847-53.

Belli, G, Cioffi, L, Fantini, C, D'Agostino, A, form more liver resections and popularize Russo, G, 2009: Laparoscopic redo surgery for recurrent hepatocellular carcinoma in cirrhotic patients: Feasibility, safety, and results. Surg. Endosc. 23, 8:1807-11.

Bhardwaj, N, Strickland, AD, Ahmad, F, et al, 2010: Microwave ablation for unresectable hepatic tumours: clinical results using a novel microwave probe and generator. Eur. J. Surg. Oncol. 36:264-8.

Bruix, J, Sherman, M, Llovet, JM, et al, 2001: Clinical management of hepatocellular carcinoma. Conclusions of the Barcelona-2000 EASL conference: European Association for the Study of the Liver. J. Hepatol. 35, 3:421-30.

Christian, DJ, Khithani, A, Jeyarajah, DR, 2011: Making liver transection even safer: a novel use of microwave technology. Am. Surg. 77, 4:417-21.

Eltawil, KM, Kidd, M, Giovinazzo, F, Helmy, AH, Salem, RR, 2010: Differentiating the impact of anatomic and non-anatomic liver resection on early recurrence in patients with Hepatocellular Carcinoma. Wld. J. Surg. Oncol. 8:43-9.

Gaujoux, S, Kingham, TP, Jarnagin, WR, D'Angelica, MI, et al, 2011: Single-incision laparoscopic liver resection. Surg. Endosc. 25, 5: 1489-94.

González, HD, Figueras, J, 2009: Surgical treatment for hepatocellular carcinoma in cirrhotic patients. Guide to the selection and decision-making process in a context of multimodal strategy. Clin. Transl. Oncol. 11, 1:20-7

Habib, M, Cresswell, AB, Chandrakumaran, K, Welsh, FK, John, TG, et al, 2012: Extrahepatic versus intrahepatic hilar control for right hepatectomy: an updated experience. Dig Surg. $29,1: 18-22$.

Huang, G, Chen, X, Lau, WY, Shen, F, Wang, RY, et al, 2014: Quality of life after surgical resection compared with radiofrequency ablation for small hepatocellular carcinomas. Br. J. Surg. May 27. doi: 10.1002/bjs.9539. [Epub ahead of print]

Huang, ZQ, Xu, LN, Yang, T, Zhang, WZ, Huang, XQ, et al, 2009: Hepatic resection: an analysis of the impact of operative and perioperative factors on morbidity and mortality rates in 2008 consecutive hepatectomy cases. Chin. Med. J. (Engl). 122, 19:2268-77.

Iannitti, DA, Martin, RC, Simon, CJ, et al, 2007: Hepatic tumor ablation with clustered microwave antennae: the US Phase II trial. HPB (Oxford) 9:120-4. 
Imura, S, Shimada, M, Utsunomiya, T, Morine, Y, Ikemoto, T, et al, 2012: Ultrasound-guided microwave coagulation assists anatomical hepatic resection. Surg. Today 42, 1: 35-40.

Jagad, RB, Koshariya, M, Kawamoto, J, et al, 2008: Laparoscopic microwave ablation of liver tumors: our experience. Hepatogastroenterology 55:27-32.

Matsumata, T, Kanematsu, T, Shirabe, K, Yamagata, M, Utsunomiya, T, et al, 1991: Modified technique of Pringle's maneuver in resection of the liver. Surg. Gynecol. Obstet. 172, 3:245-6.

Min, YW, Lee, JH, Gwak, GY, Paik, YH, Lee, JH, et al, 2014: Long-term survival after surgical resection for huge hepatocellular carcinoma: comparison with transarterial chemoembolization after propensity score matching. J. Gastroenterol. Hepatol. 29, 5:1043-8.

Nasr, M, Nafee, N, Saad, H, Kazem, A, 2014: Improved antitumor activity and reduced cardiotoxicity of epirubicin using hepatocyte-targeted nanoparticles combined with tocotrienols against hepatocellular carcinoma in mice. Eur. J. Pharm. Biopharm. 2014 May 6. pii: S0939-6411(14) 00133-7. doi: 10.1016/j.ejpb.2014.04.016.

Pawella, LM, Hashani, M, Eiteneuer, E, Renner, M, Bartenschlager, R, et al, 2014: Perili- pin discerns chronic from acute hepatocellular steatosis. J. Hepatol. 60, 3:633-42

Percivale, A, Griseri, G, Gastaldo, A, Benasso, M, Pellicci, R, 2012: Microwave assisted liver resection: clinical feasibility study and preliminary results. Minerva Chir. 67, 5:415-20.

Reuter, NP, Martin, RC, $2^{\text {nd }}$, 2009: Microwave energy as a pre-coagulative device to assist in hepatic resection. Ann. Surg. Oncol. 16, 11: 3057-63.

Tanaka, K, Shimada, H, Matsumoto, C, Matsuo, K, Nagano, Y, et al, 2008: Anatomic versus limited nonanatomic resection for solitary hepatocellular carcinoma. Surgery 143, 5:60715.

Tarantino, G, Citro, V, Esposito, P, Giaquinto, S, de Leone, A, 2009: Blood ammonia levels in liver cirrhosis: a clue for the presence of portosystemic collateral veins. BMC Gastroenterol. 2009, Mar 17;9:21. doi: 10.1186/1471230X-9-21.

Teoh, NC, Farrell, GC, 2003: Hepatic ischemia reperfusion injury: pathogenic mechanisms and basis for hepatoprotection. J. Gastroenterol. Hepatol. 18, 8:891-902.

Teoh, NC, Farrell, GC, 2003: Hepatic ischemia reperfusion injury: pathogenic mechanisms and basis for hepatoprotection. $\mathrm{J} /$ Gastroenterol. Hepatol. 18, 8:891-902

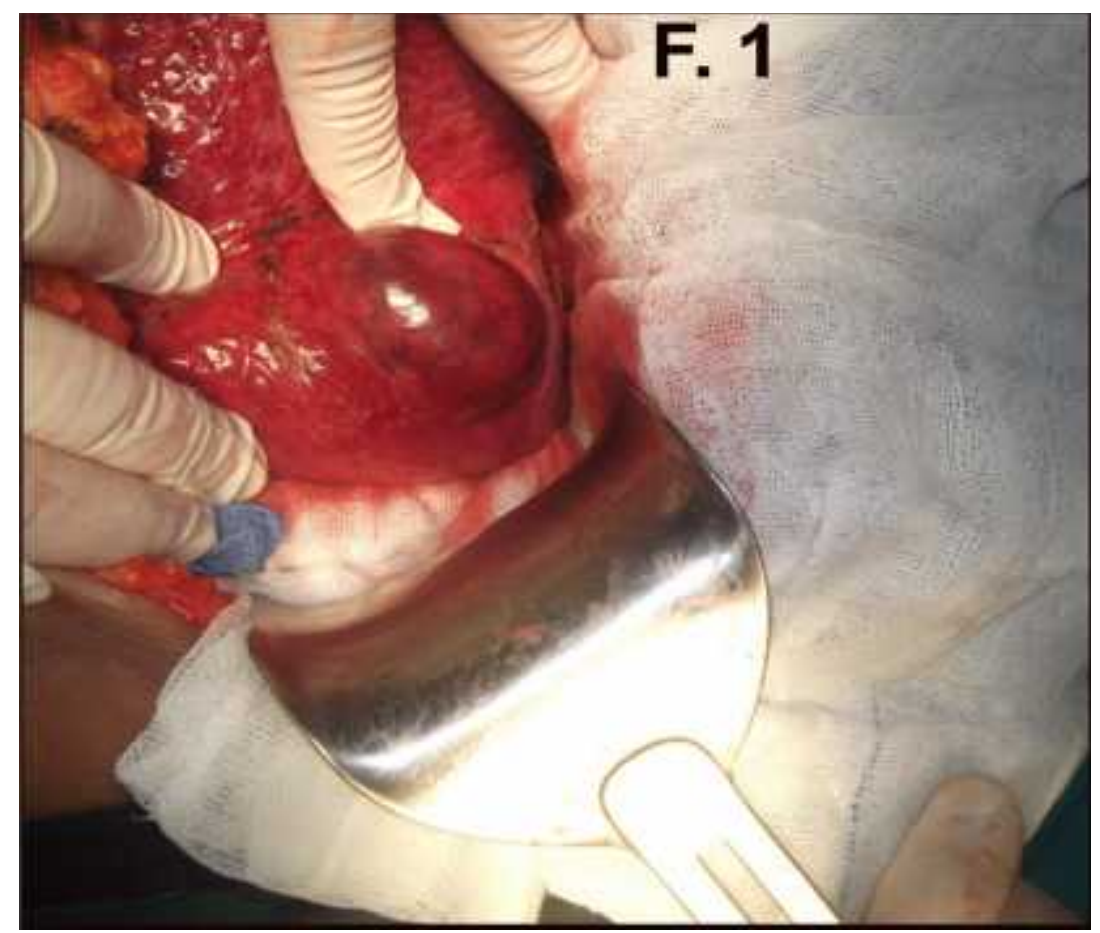

Figure 1: Identification of resection margin by Diathermy 


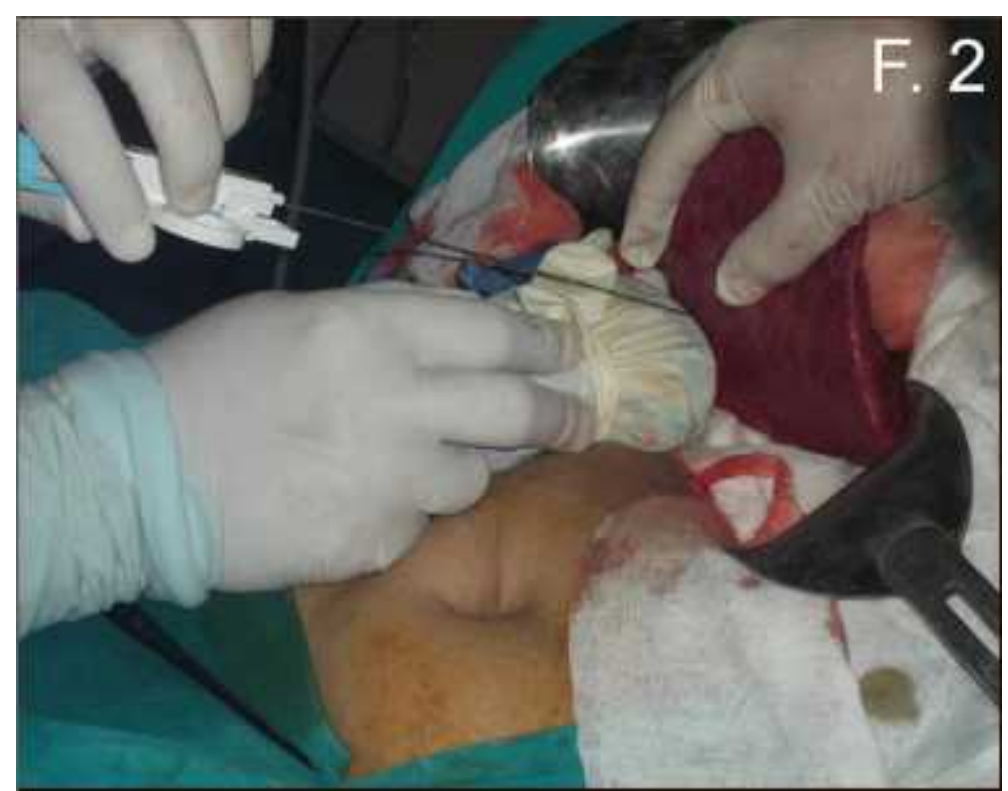

Figure 2: U/S guidance of Needle insertion

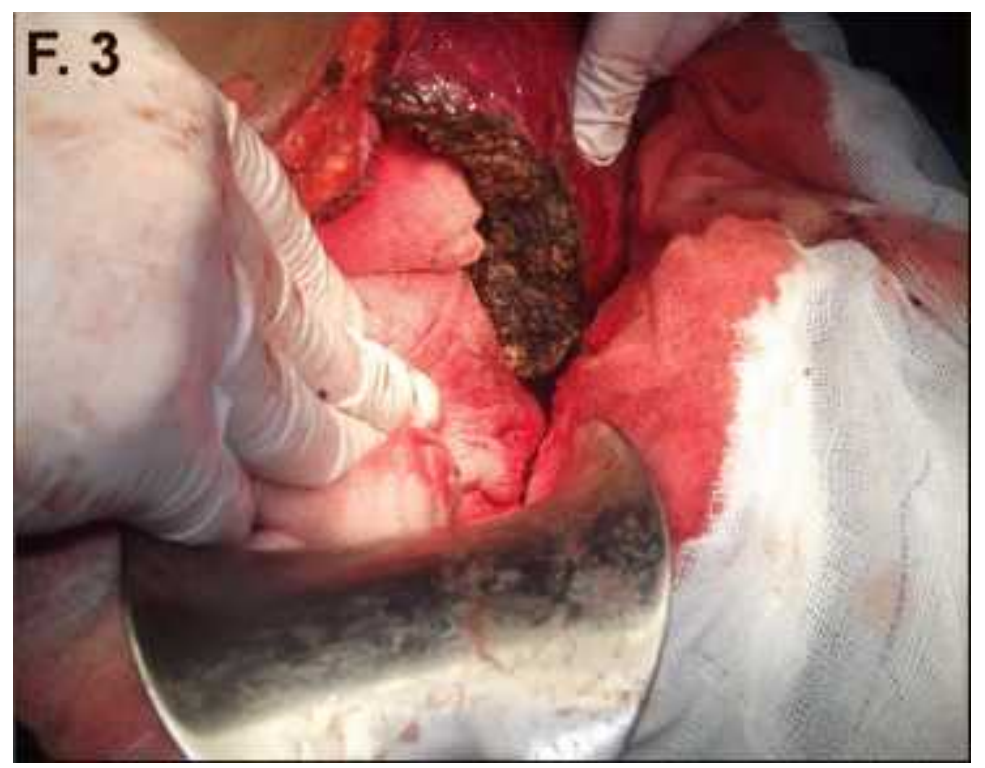

Figure 3: Resection margin hemostasis

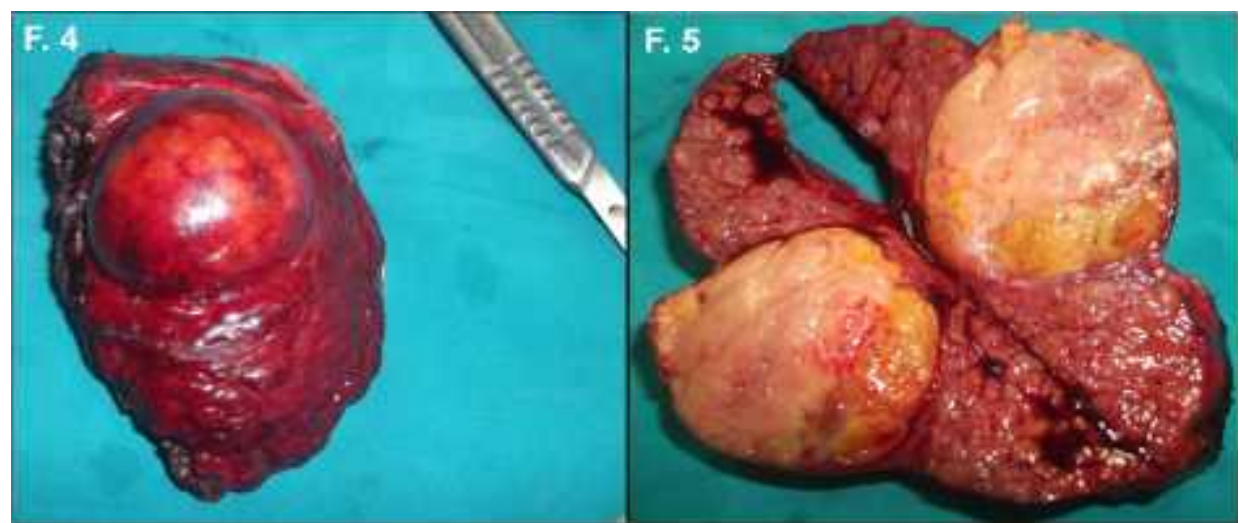

Figure 4: Specimen after resection Figure 5: Specimen opened with good resection margin 